\title{
A Disconfirmation of the Quantitative Identity- Quantitative Equivalence Sequence
}

\author{
Scott A. Miller \\ University of Michigan
}

\begin{abstract}
Sixty-four kindergarten children received tests of quantitative identity and quantitative equivalence for the conservations of number and continuous quantity. Two types of identity trials were included: a standard version using a single stimulus, and a modified version which paralleled the equivalence task in its use of two stimuli. In addition, half of the children were asked two questions on each trial (one preceding and one following the transformation), whereas half were asked only the post-transformation question. Neither the number of stimuli used nor the number of questions asked had any effect on performance. In contrast to some previous reports. tests of quantitative identity were no easier than tests of quantitative equivalence. It was concluded that the identity-equivalence décalage, if it exists at all, is less important than previous authors have claimed.
\end{abstract}

In a typical conservation-of-number lask (e.g., Piaget, 1952), the child is shown two rows with equal numbers of objects, lined up in one-to-one correspondence. After the child has agreed to the equality. one of the rows is spread out, creating the appearance of unequal numbers. The child is then asked whether the rows still contain the same number of objects.

Elkind (1967) was the first to note that this task actually taps two somewhat distinct kinds of knowledge. The child who answers correctly has presumably realized that the number of objects in the transformed row has not been altered by the change in perceptual appearance. This understanding, labeled quantitative identity, is the ability stressed in Piaget's theory. In the typical test, however, the child is never asked directly about the identity of the transformed row; instead, the question concerns the relative number of objects in the two rows. The realization that the two rows still contain equal numbers is referred to as quantitative equivalence.

The point of Elkind's distinction is that an understanding of identity would seem to be a necessary but not sufficient basis for solution of the equivalence problem. What must be added to the recognition of identity is a

This study was supported by funds provided by the Department of Psychology, University of Michigan. I am grateful to Tammy Nelson for her excellent assistance with the testing, and to the staff and children of Isbister Elementary School, Plymouth. Michigan. for their generous cooperation. Requests for reprints should be sent to Scott A. Miller, Department of Psychology, University of Florida, Gainesville. Fla. 32611. 
kind of transitive inference: The two rows were the same to start with, the transformed row still has the same number of objects as it did at the start (this is the identity component), therefore the transformed row still has the same number as the nontransformed row (the equivalence component). Given the need for this additional deductive step, it may be that the equivalence task underestimates the child's actual ability to conserve. Perhaps children would perform better if they were given a direct test of quantitative identity. It should be noted that the issue here is not simply the establishment of accurate age norms for the mastery of conservation. Rather, the question of when conservation first emerges is related to central Piagetian claims concerning sequences and concurrences in the development of concepts.

Since Elkind's article, there have been eleven reported studies comparing the relative difficulty of identity tasks and equivalence tasks in samples of normal children. The results have been mixed. In seven studies there has been some (although often weak) support for the hypothesis that identity develops earlier than equivalence (Elkind \& Schoenfeld, 1972; Hooper, 1969a, b; Papalia \& Hooper, 1971; Rybash, Roodin, \& Sullivan, 1975; Schwartz \& Scholnick, 1970; Toniolo \& Hooper, Note 1). Four other studies reported no differences between the two concepts (Koshinsky \& Hall, 1973; Moynahan \& Glick, 1972; Murray, 1970; Northman \& Gruen, 1970).

In a recent review, Brainerd and Hooper (1975) conclude that studies which support the identity-equivalence sequence give a more accurate picture of development than those which fail to support it. The latter set of studies is said to suffer from two methodological flaws: the requirement of a verbal explanation as part of the criterion for conservation, and a concentration on subjects old enough to pass any version of the conservation test. As discussed in more detail elsewhere (Miller, in press), neither of these variables in fact provides a very satisfactory explanation for the discrepant results. The choice of a judgments-only or a judgments-plus-explanations criterion appears to have little effect on the comparison of identity and equivalence. And in none of the nonsupportive studies is there any suggestion that ceiling effects have masked a true identity-equivalence sequence. In addition, at least two of the supportive studies have methodological problems which Brainerd and Hooper fail to note. Specifically, the nonverbal assessment technique in the Schwartz and Scholnick (1970) study is appropriate only for equivalence, not for identity, and the Toniolo and Hooper (Note 1) study is rendered suspect by the ambiguous wording of the equivalence question (a wording that is incorrectly reported by Brainerd and Hooper, 1975; see Miller, in press, for a fuller discussion).

The question of whether identity precedes equivalence in development thus remains unresolved. Also unresolved is the question of why the two tasks differ in difficulty, if in fact they do. Theorizing to date has focused on 
Elkind's (1967) contention that the equivalence task involves an additional deductive step that is not required for identity. There are, however, at least two other potentially important ways in which equivalence problems differ from identity problems. First, the perceptual pull toward nonconservation is almost certainly greater for equivalence than for identity. In the former task the child is confronted with two perceptually discrepant stimuli at the time of the conservation question, whereas in the latter task only one stimulus is present. The nonconservation illusion on an identity trial is dependent on the child's comparison of the transformed stimulus with his memory of the stimulus prior to the transformation. It may be that the weaker illusion on the identity problem is what accounts for its greater ease.

The other difference between the two paradigms concerns the number of questions asked. An identity test requires just one question, asked after the stimulus has been transformed. An equivalence test typically involves two questions: a pretransformation question to establish the initial equality of the two stimuli, and the post-transformation conservation question. Rose and Blank (1974) have recently reported that children perform better on the equivalence test if the initial question is omitted and only the final conservation question is asked. Their explanation for this finding is that children who are asked two questions interpret the second question as a sign that they should change their original answer. Since the original judgment was one of "same," the result would be a bias toward nonconservation. This effect is great enough in the Rose and Blank study to suggest that it alone might account for the reported differences between identity and equivalence.

In the present study, possible effects of the degree of perceptual illusion are examined through the inclusion of identity trials which use two stimuli and which are therefore perceptually identical to the equivalence trials. All children receive trials of this sort as well as standard identity and equivalence trials. Possible effects of the number of questions are examined through the assignment of children to one of two experimental conditions. In one condition only one question is asked on all trials (both identity and cquivalence), whereas in the second condition two questions are asked on all trials. The concepts studied are conservation of number and conservation of continuous quantity.

\section{METHOD}

\section{Subjects}

The subjects were 64 kindergarten children (mean age $=5$ years, 11 months). drawn from a predominantly lower-middle-class school in southeastern Michigan. Three additional children were rejected because of failure to pass the verbal pretest. The final sample included 16 boys and 16 girls in each of the two experimental conditions. All of the children were white. 


\section{Procedure}

The experimenter was a white, college-aged female. As a guard against possible experimenter bias, the tester was not informed of any of the hypotheses underlying the study.

Verbal pretest. The session began with a verbal pretest designed to assure that the child understood the terms that would be used on the conservation trials. The first pretest trial dealt with number and the second with quantity. Stimuli for the first trial were $4 \frac{1}{2} 2-\mathrm{cm}$ cardboard squares; stimuli for the second trial were wooden blocks. On both trials, the child was asked to compare a given stimulus with stimuli which were equal to it on the quantitative dimension in question, greater than it. and less than it. For example, the number trial required the child to compare a group of two squares successively with groups of two squares, three squares, and one square. The form of the question was the same as that on the later conservation trials: "Are there the same number of squares here as there are here, or are there more squares here than there are here, or are there less squares here than there are here?" (On half the trials the order of the phrases was "more," "less," "same.")

Any child who failed a question was given correction, followed by a second trial (using different stimuli from those on the first trial) for the quantitative dimension on which he had failed. Children who failed the sccond trial for either of the two dimensions were eliminated from the study.

Conservation test. Each child received three trials for conservation of number and three for conservation of liquid quantity. The order of the number and quantity trials was balanced across subjects. Within each concept the three trials consisted of an identity problem with one stimulus, an identity problem with two stimuli, and an equivalence problem. The equivalence problem came first for half the subjects and last for the other half. The order of the two forms of identity problem was balanced across subjects. For a particular subject, the ordering of the three types of problem was the same for number as for quantity.

Half of the children were assigned to the One-Question experimental condition and half to the Two-Questions condition. The procedure is described with respect to the One-Question condition, after which the differences for the Two-Questions condition are noted.

The stimuli for the number trials were 14 red poker chips, a $54 \times 70-\mathrm{cm}$ yellow posterboard, and two $4.5 \times 70-\mathrm{cm}$ strips of white posterboard which could be laid horizontally across the center of the yellow board. The strips of white board were marked off with two vertical ink lines $36 \mathrm{~cm}$ apart. These lines served to mark the endpoints for the rows of chips. On trials with one row only one strip was used; on trials with two rows both strips were used.

All trials began with the experimenter saying, "Watch what I do." The 
one-stimulus identity trial proceeded with the experimenter laying out seven chips on the white strip. The chips were left in place for a few seconds, after which the experimenter simultaneously moved the chips off the white board to the yellow and either spread them or bunched them. (The two transformations alternated from trial to trial and appeared equally often for each of the three trials.) The child was then asked, "Are there the same number of chips here [pointing to the transformed row] as there were here [pointing to the white strip], or are there more chips here than there were here, or are there less chips here than there were here?" (On half the trials the order of the phrases was "more," "less," "same.") Following his judgment, the child was asked, "Why do you think so?"

The two-stimuli identity trial began with the experimenter laying out two rows of seven chips, lined up in one-to-one correspondence on the two white strips. After a delay of a few seconds, one of the rows was transformed in the manner described above and the same question was asked. Again, each phrase of the question was accompanied by gestures indicating which row was being referred to.

The equivalence trial was identical in physical arrangement to the two-stimuli trial just described. In this case, however, the question concerned the equivalence of the two rows: "Are there the same number of chips here [pointing to the transformed row] as there are here [pointing to the nontransformed row], or are there more chips here than there are here, or are there less chips here than there are here?" On both this and the preceding trial, the white strips were moved apart prior to the transformation and the transformed row ended up between the two strips. Thus, on both trials the stimuli that the child was asked about were adjacent at the time of the question.

The stimuli for the liquid quantity trials consisted of colored water and four plastic beakers. Two of the beakers (the standards) were $14 \mathrm{~cm}$ tall and $10.5 \mathrm{~cm}$ wide. The remaining beakers were $28 \mathrm{~cm}$ tall by $7.5 \mathrm{~cm}$ wide and 7.5 $\mathrm{cm}$ tall by $15 \mathrm{~cm}$ wide. The two standard beakers contained water-level markings on the side that was presented to the child. The water was always poured to the top mark, $1.3 \mathrm{~cm}$ from the top of the beaker.

The one-stimulus identity trial began with the experimenter's "Watch what I do," followed by the filling of one of the standard containers. After a pause, the water was poured into either the taller or the shorter beaker (again, the two transformations were used equally often on each of the three trials). The wording of the conservation question was the same as that for number, with the substitution of "same amount of water" for "same number of chips."

As on the number problems, the two-stimuli identity and the equivalence trials were physically identical. Each began with the filling of two standard containers to the same level, followed by the emptying of one into either the taller or the shorter beaker. The trials differed in whether an identity or an equivalence question was asked. The taller or shorter bcaker was always 
placed between the two standards at the time of the conservation question, thus permitting a comparison of adjacent beakers for both identity and equivalence.

The basic difference between the procedure just described and the Two-Questions condition was that children under the latter condition were asked two questions on each trial, one preceding and one following the transformation. On the four trials which began with two equal stimuli (i.e., the equivalence and two-stimuli identity trials), the first question was the standard one to establish the initial equality of the stimuli. The wording was the same as that for the final conservation question. If the child did not agree to the initial equality, the stimuli were adjusted until agreement was reached.

The two one-stimulus identity trials did not present a pair of equal stimuli about which the child could be questioned prior to the transformation. The initial question on these trials was made possible through movement of the stimuli from the standard starting state to an identical second standard. Thus, for number, the row of seven chips was simply moved from one white strip to an identical arrangement on the other white strip, and the conservation question was asked. Similarly, for water, the contents of one standard were emptied into an identical second standard. After the child's response to the first question, the conservation transformation was performed and the final question was asked.

\section{Scoring}

Two judges independently rated the explanations that accompanied correct conservation judgments. Explanations from the following categories were considered adequate: addition/subtraction, inversion, compensation, previous equality, irrelevancy of transformation, and (for number only) one-to-one correspondence and counting or reference to absolute number. The percentage of agreement with respect to adequacy of explanation was $96 \%$.

\section{RESULTS}

Performance on the conservation trials is summarized in Table 1. The results are presented in terms of two criteria: proportion of trials with correct judgments, and proportion of trials with correct judgments accompanied by adequate explanations. Analyses were carried out using both criteria.

The design of the study included the between-subject factors of condition (One Question vs Two Questions) and sex and the within-subject factors of concept (number vs quantity) and type of trial. The dichotomous nature of performance at the level of specific trial precluded a single overall analysis of variance. Consequently, two main sets of analyses were performed: 
TABLE 1

Proportion of Correct ANSWers and Correct ANSWERS with ADEQuate Explanations

\begin{tabular}{|c|c|c|c|c|c|c|}
\hline & \multicolumn{2}{|c|}{ One-question condition } & \multicolumn{2}{|c|}{ Two-questions condition } & \multicolumn{2}{|c|}{ Both conditions combined } \\
\hline & $\begin{array}{l}\text { Correct } \\
\text { answers }\end{array}$ & $\begin{array}{c}\text { With } \\
\text { adequate } \\
\text { explanations }\end{array}$ & $\begin{array}{l}\text { Corred } \\
\text { answers }\end{array}$ & $\begin{array}{c}\text { With } \\
\text { adequate } \\
\text { explanutions }\end{array}$ & $\begin{array}{l}\text { Correct } \\
\text { answers }\end{array}$ & $\begin{array}{c}\text { With } \\
\text { adequale } \\
\text { explanations }\end{array}$ \\
\hline \multicolumn{7}{|l|}{ Number } \\
\hline One-stimulus identity & .44 & .34 & hth & .4 & .15 & 3.8 \\
\hline Two-stimuli identity & .41 & .34 & 63 & .41 & .52 & $.3 \times$ \\
\hline Equivalence & .47 & .44 &.+4 & .34 & 4.5 & .89 \\
\hline All trials & .44 & $.3 x$ &.$\$ 7$ & .39 & 51 & $.3 x$ \\
\hline \multicolumn{7}{|l|}{ Continuous quantity } \\
\hline One-stimulus identity & .41 & .25 & in & $.2 x$ & .48 & .27 \\
\hline Two-stimuli identity & .47 & $.3 x$ & .41 & .31 & 44 & .34 \\
\hline Equivalence & .34 & .31 & .41 & .38 & .39 & .34 \\
\hline All trials & +1 & .31 & .47 & .32 & .4 & .32 \\
\hline \multicolumn{7}{|l|}{ Both ioncepts } \\
\hline One-stimulus identity & .42 & .30 & .61 & .34 & .52 & .32 \\
\hline Two-stimuli identity & .44 & .36 & $\therefore 2$ & .36 & $.4 k$ & 36 \\
\hline Equivalence & 41 & .38 & +4 & .36 & .42 & .37 \\
\hline All trials & 42 & .34 & 52 & 35 & .47 & .35 \\
\hline
\end{tabular}

$2 \times 2 \times 2$ analyses of variance on the factors of condition, sex, and concept, and a series of Cochran $Q$ tests to examine the effects of type of trial.

The analyses of variance revealed no statistically significant effects as a function of condition, sex, concept, or the interactions among these factors (all $p$ 's $>.10$ ). As Table 1 indicates, correct judgments were slightly more frequent under the Two-Questions condition than under the One-Question condition, a direction of difference opposite to that obtained by Rose and Blank (1974). The difference, however, was well short of statistical significance $[F(1,60=1.12, p>.25]$. Correct answers were also more frequent on number trials than on quantity trials. but again the difference did not approach significance $[F(1.60=1.86, p>.10]$. Finally, the performance of the two sexes was virtually identical: $46 \%$ correct answers for boys, $48 \%$ for girls.

Comparisons among the three types of trial were carried out both for each concept separately and for both concepts combined, yielding a total of six Cochran Q tests (i.e., tests for number, quantity, and number-quantity combined, using both a judgments-only and a judgments-plus-explanations criterion). For the combined tests, the children's performance was categorized as either passing both instances of a given type of trial or not passing both instances. On none of the six tests did the differences among trials approach statistical significance (the largest $\chi^{2}$ was 2.67, for correct judgments on the number trials). Thus, there was no evidence that 
the number of stimuli with which the child had to deal affected the difficulty of the identity task. Nor was there any evidence that a mastery of identity developed earlier than a mastery of equivalence.

\section{DISCUSSION}

This study tested two possible explanations for reported differences between quantitative identity and quantitative equivalence. Equivalence tasks have typically presented two perceptually discrepant stimuli at the time of the conservation question, whereas identity tasks have presented only one stimulus. The current results indicate that the number of stimuli does not affect the difficulty of the task. This finding is in accord with data from studies by Hooper (1969b) and Koshinsky and Hall (1973), both of which used screening of the nontransformed standard to equate the perceptual demands of identity and equivalence tasks. Thus, although most comparisons of identity and equivalence have failed to equate the degree of perceptual illusion, the available evidence suggests that this variable is not an important contributor to differences in difficulty.

The second variable examined was the number of questions asked. Rose and Blank (1974) had reported that a one-question version of the equivalence task was considerably easier than the standard two-questions version. This result was not replicated here; in fact, the mean differences were in the opposite direction. The present results offer no support for the hypothesis that asking a second question biases the child toward a nonconservation judgment. It follows that there is also no support for the hypothesis that such a bias could account for reported differences between identity and equivalence.

The discussion thus far has considered two possible explanations for differences between identity and equivalence. The major finding of the study, however, was that no such differences existed. Neither within nor across conditions was there any statistically significant evidence to indicate that identity problems were easier than equivalence problems. This conclusion also holds if the analysis is limited to trials which presented these problems in their typical form, i.e., identity trials with one stimulus and one question, and equivalence trials with two questions. The former sort of trial yielded $42 \%$ correct responding; the latter sort, $44 \%$.

As noted earlier, Brainerd and Hooper (1975) propose two explanations for apparent disconfirmations of the identity-equivalence sequence: testing of subjects too developmentally mature to show the sequence, and use of a judgments-plus-explanations criterion. Neither of these explanations can account for the present results. The figures in Table 1 indicate clearly that ceiling effects were not a problem. The sample was, in fact, at an ideal level for finding differences among trials. if the trials in fact differed in difficulty. Of the 64 subjects, 38 were inconsistent in their performance, 
neither passing nor failing all of the trials. There was, then, ample variability in performance; the variability, however, did not relate systematically to the nature of the trial.

The choice of a particular criterion for conservation proved to be unimportant. It is true that mean differences by the judgments-only criterion favored the identity tasks, whereas the differences disappeared or were reversed with judgments plus explanations as the criterion. Obviously, children found it more difficult to explain their correct judgments on identity trials than on equivalence trials. By neither criterion, however, did the differences between identity and equivalence approach statistical significance.

The conclusion from Brainerd and Hooper's (1975) review was that the use of the equivalence paradigm may seriously underestimate the child's ability to conscrve. This concern appears to be overstated. It is probably the case that some children do find identity tasks easier than equivalence tasks; there are, after all, logical, perceptual, and linguistic bases for such an effect. Even in studies which report a difference, however, the difference is often quite small. There are, moreover, a substantial number of studies which find no difference at all. A reasonable conclusion is that for most children identity and equivalence are contemporaneous developmental achievements.

\section{REFERENCES}

Brainerd, C. J.. \& Hooper, F. H. A methodological analysis of developmental studies of identity conservation and equivalence conservation. Psychological Bulletin, $1975,82$. $725-737$.

Elkind, D. Piaget's conservation problems. Child Development, 1967, 38, 15-27.

Elkind, D., \& Schoenfeld, E. Identity and equivalence conservation at two age levels. Developmental Psychology, 1972, 6, 529-533.

Hooper, F. H. The Appalachian child's intellectual capabilities-Deprivation or diversity? Journal of Negro Education, 1969a, 38, 224-235.

Hooper, F. H. Piaget's conservation tasks: The logical and developmental priority of identity conservation. Journal of Experimental Child Psychology. 1969b. 8, 234-249.

Koshinsky, C. \& \& Hall. A. E. The developmental relationship between identity and equivalence conservation. Journal of Experimental Child Psychology, 1973, 15, $419-424$.

Miller, S. A. Identity conservation and equivalence conservation: A critique of Brainerd and Hooper's analysis. Psychological Bulletin. in press.

Moynahan, E., \& Glick, J. Relation between identity conservation and equivalence conservation within four conceptual domains. Developmental Psychology, 1972, 6, $247-251$

Murray, F. B. Stimulus mode and the conservation of weight and number. Joumal of Educational Psychology, 1970, 61, 287-291.

Northman, J. E., \& Gruen, G. E. Relationship between identity and equivalence conservation. Developmental Psychology, 1970. 2, 311.

Papalia, D. E., \& Hooper, F. H. A developmental comparison of identity and equivalence conservations. Journal of Experimental Child Psychology. 1971, 12, 347-361.

Piaget. J. The child's conception of number. New York: Humanities Press, 1952. 
Rose, S. A., \& Blank, M. The potency of context in children's cognition: An illustration through conservation. Child Development, 1974, 45, 499-502.

Rybash, J. M., Roodin, P. A., \& Sullivan, L. F. The effects of a memory aid on three types of conservation judgments. Journal of Experimental Child Psychology, 1975, 19, $358-370$.

Schwartz, M. M., \& Scholnick. E. K. Scalogram analysis of logical and conceptual components of conservation of discontinuous quantity. Child Development, 1970, 41, $695-705$.

\section{REFERENCE NOTE}

1. Toniolo, T. A., \& Hooper. F. H. Micro-analysis of logical reasoning relationships: Conservation and transitivity (Tech. Rep. No. 326). Madison: Wisconsin Research and Development Center for Cognitive Learning. April 1975.

ReCEIVED: July 29, 1976; REVISED: September 27, 1976 